\title{
Genome Sequence Data of MAT1-1 and MAT1-2 Idiomorphs from Verticillium dahliae
}

Ya-duo Zhang, ${ }^{1}$ Yuan-Yuan Zhang, ${ }^{2}$ Jie-Yin Chen, ${ }^{1}$ Jin-Qun Huang, ${ }^{3}$ Jian Zhang, ${ }^{2}$ Lin Liu, ${ }^{2}$ Dan Wang, ${ }^{1}$ Jun Zhao, ${ }^{2}$ Jian Song, ${ }^{1}$ Ran Li, ${ }^{1}$ Lin Yang, ${ }^{3}$ Zhi-Qiang Kong, ${ }^{1}$, Steven J. Klosterman, ${ }^{4}$ Krishna V. Subbarao, ${ }^{5, \dagger}$ Xiao-Feng Dai, ${ }^{1, \dagger}$ and Dan-dan Zhang ${ }^{1, \dagger}$

${ }^{1}$ State Key Laboratory for Biology of Plant Diseases and Insect Pests, Institute of Plant Protection, Chinese Academy of Agricultural Sciences, Beijing, 100193, China

${ }^{2}$ College of Horticulture and Plant Protection, Inner Mongolia Agricultural University, Hohhot, 010018, China

${ }^{3}$ BGI-Genomics, BGI-Shenzhen, Shenzhen, 518083, China

${ }^{4}$ U.S. Department of Agriculture, Agricultural Research Service, Crop Improvement and Protection Research Unit, Salinas, CA, U.S.A.

${ }^{5}$ Department of Plant Pathology, University of California, Davis, c/o U.S. Agricultural Research Station, Salinas, CA, U.S.A.

\section{Abstract}

Though Verticillium dahliae is an asexually reproducing fungus, it is considered heterothallic owing to the presence of only one of the two mating-type idiomorphs (MAT1-1 or MAT1-2) in individual isolates. But sexual reproduction has never been observed either in nature or in the laboratory. All of the genomic information in the literature thus far has therefore come from studies on isolates carrying only the MAT1-2 idiomorph. Herein, we sequenced and compared high-quality reference genomes of MAT1-1 strain S011 and MAT1-2 strain S023 obtained from the same sunflower field. The two genomic sequences displayed high synteny, and encoded similar number genes, a similarity especially notable among pathogenicity-related genes. Homolog analysis between these two genomes revealed that $80 \%$ of encoded genes are highly conserved ( $95 \%$ identity and coverage), but only $20 \%$ of the single copy genes were identical. These novel genome resources will support the analysis of the structure and function of the two idiomorphs and provide valuable tools to elucidate the evolution and potential mechanisms of sexual reproduction in V. dahliae.

Sexual reproduction in heterothallic ascomycetes is controlled by alternative forms of the mating-type locus MAT1-1 and MAT1-2 (Coppin et al. 1997; Dyer et al. 2016; Pöggeler 2001; Turgeon and Yoder 2000). MAT1-1 encodes an alpha-box transcription factor and MAT1-2, a high mobility group (HMG) transcription factor domain. These transcription factors target pheromone precursor and receptor genes that mediate cell attraction and fusion during sexual reproduction but also affect other cellular processes (Becker et al. 2015; Keszthelyi et al. 2007). The alpha1 gene present at MAT1-1 is designated 'MAT1-1-1', and the HMG box gene at MAT1-2 is referred to as MAT1-2-1. In addition, other genes are present at MAT depending on the species, and these include MAT1-1-2, MAT1-1-3, and MAT1-1-4 at the MAT1-1 idiomorph and similarly, MAT1-2-2, MAT1-2-3, and MAT1-2-4 at the MAT1-2 idiomorph (Keszthelyi et al. 2007; Klix et al. 2010; Turgeon and Yoder 2000).

${ }^{\dagger}$ Corresponding authors: K. V. Subbarao; kvsubbarao@ucdavis.edu,

X.-F. Dai; daixiaofeng_caas@126.com, and D.-D. Zhang; zhangdandan@caas.cn

Current address for Y.-Y. Zhang: Institute of Grassland Research, Chinese Academy of Agricultural Sciences, Hohhot 010010, China.

First, second, third, and fourth authors contributed equally to this work.

The author(s) declare no conflict of interest.

Accepted for publication 2 March 2021.
Funding

The study was sponsored by National Key Research and Development Program of China (2018YFE0112500 and 2017YFD0201900), the Elite Youth Program CAAS to J.-Y. Chen, the Fundamental Research Funds for Central Nonprofit Scientific Institution (Y2018PT70), the Agricultural Science and Technology Innovation Program grant to X.-F. Dai, the China Agricultural Research System (CARS-14), and the National Natural Science Foundation of China (31870138, 31970142, and 31972228).

\section{Keywords}

analytical and theoretical plant pathology, bacteriology, bioinformatics, disease control and pest management, evolution bacterial pathogens, fungal pathogens, mycology 
$V$. dahliae is considered an asexual pathogen. The most intuitive evidence for this is the lack of sexual structures in either natural or laboratory conditions (Short et al. 2014). Several population genetic studies also support clonal propagation in V. dahliae (Atallah et al. 2011; Baroudy et al. 2019; Milgroom et al. 2014, 2016; Rafiei et al. 2018; Short et al. 2015). However, V. dahliae has preserved two different mating type loci (MAT1-1 and MAT1-2) and is heterothallic (Usami et al. 2008, 2009). Interestingly, the mating type frequencies in $V$. dahliae in nature is heavily skewed toward the MAT1-2 idiomorph (Milgroom et al. 2014; Short et al. 2015; Usami et al. 2008). Comparison of genome structures revealed extensive chromosomal rearrangements among the different $V$. dahliae genomes (Chen et al. 2018; de Jonge et al. 2013), which are expected to interfere in the correct pairing of homologous chromosomes during meiosis (Kistler and Miao 1992). Therefore, $V$. dahliae is mainly considered to be an asexual species that propagates clonally (Seidl and Thomma 2014).

Several recent studies, however, have speculated outcrossing in $V$. dahliae, a feature that was likely in ancestral species or an extant ability that conferred cryptic sexual reproduction (Atallah et al. 2010, 2012; Inderbitzin et al. 2013; Milgroom et al. 2014; Short et al. 2014; Usami et al. 2009). As noted above, V. dahliae isolates contain either the MAT1-1 or MAT1-2 idiomorph, and the sex-related genes are conserved, active, and are under purifying selection (Short et al. 2014). Moreover, several V. dahliae recombinant haplotypes are likely to have arisen by recombination between closely related lineages (Milgroom et al. 2014). In addition, although the parasexuality was speculated as the likely origin of the diploid V. Iongisporum (Karapapa et al. 1997), it has been discounted for lack of evidence. However, recent studies have clearly demonstrated the origin of this species via hybridization between $V$. dahliae and an unknown species (Depotter et al. 2016; Inderbitzin et al. 2011a, b). The stability of V. longisporum as a hybrid suggests that interspecific hyphal fusion followed by nuclear fusion as more plausible than parasexual processes for its origin (Inderbitzin et al. 2011b).

At present, more than $20 \mathrm{~V}$. dahliae genome sequences ( 35 Mb each) have been publicly released (https://www.ncbi.nlm.nih.gov/genome/genomes/832) and all of them carry the MAT1-2 idiomorph, except the whole genome sequences of $85 \mathrm{~S}$ strain (GenBank assembly accession: GCA_004798885.1,39 of scaffolds) and Vd39 strain (GCA_011032905.1, 1578 of scaffolds) isolated from sunflower in France and Germany, respectively, that carry the MAT1-1 idiomorph (Depotter et al. 2019). Therefore, the relative dearth of genome information for the MAT1-1 idiomorph has limited our understanding and research into the sexual mode of reproduction in V. dahliae.

The distribution of the two MAT idiomorphs in relative equilibrium was observed in the sunflower and potato cropping systems in Inner Mongolia, China (Verticilli-Omics, https://db. cngb.org/Verticilli-Omics/), which suggested that MAT1-1 idiomorph has adapted and expanded under some environments in China, resulting in enhanced risk of sexual reproduction in $V$. dahliae. To determine the genomic basis of potential sexual mating and to facilitate the study of this phenomenon, we sequenced the genomes of $V$. dahliae strains S011 and S023 isolated from a sunflower field in Inner Mongolia, carrying the MAT1-1-1 and MAT1-2-1 sequences, respectively.

The high-quality genomic DNA of these two strains was extracted for genome sequencing using a Quick-DNA Fungal/Bacterial Midiprep Kit (Zymo Research, Orange, CA, U.S.A.). For whole-genome sequencing, two libraries with insertion sizes of $20 \mathrm{~kb}$ and $400 \mathrm{bp}$ were constructed using SMRTbell Template Prep Kits (Pacific Biosciences, Menlo Park, CA, U.S.A.) and Illumina TruSeq Nano DNA Library Prep Kits (Illumina, San Diego, CA, U.S.A.), and then sequenced using PacBio Sequel and Illumina Hiseq 2000 platforms, respectively. The adapter sequence, low-quality reads ( $<\mathrm{Q} 30)$, and $N$ bases of PacBio and Illumina data were filtered. The assembly procedure was employed to assemble the genomes as described previously (Chen et al. 2020), including de novo assembly by SMARTdenovo (version 170825), extended by the SSPACE (version STANDARD-3.0) program using sequencing reads (Boetzer and Pirovano 2014), minding gap by PBjelly2 program (English et al. 2012, version 15.8.24), error correction by the variantCaller program (version 2.2.2) from the SMRTlink v4 package (Pacific Biosystems), and polished the assembled sequences by GATK (McCormick et al. 2015, version 3.4.0), SOAPsnp (version 1.05), and SOAPindel (version 1.08) programs.

The final genome assemblies of S011 and S023 consist of 12 and 13 scaffolds, for a total genome size of $37,324,492$ bp (N50 length of 4,479,799 bp) and 35,377,336 bp (N50 length of $3,798,651 \mathrm{bp}$ ), respectively. The length of the top eight assembled sequences of S011 and S023 were $34,287,596$ and $31,582,067 \mathrm{bp}$, amounting to 91.86 and $89.27 \%$ of the genome sizes, 
Table 1. Genome statistics of Verticillium dahliae strains S011 and $\mathrm{S}_{02} 3^{\mathrm{a}}$

\begin{tabular}{|c|c|c|}
\hline \multirow[b]{2}{*}{ Statistics } & \multicolumn{2}{|c|}{ Scaffold } \\
\hline & S011 (MAT1-1) & S023 (MAT1-2) \\
\hline \multicolumn{3}{|l|}{ Genome assembly } \\
\hline Total number & 12 & 13 \\
\hline Total length (bp) & $37,324,442$ & $35,377,336$ \\
\hline Gap number (bp) & 25 & 25 \\
\hline Average length (bp) & $3,732,449$ & $2,721,334$ \\
\hline N50 length (bp) & $4,479,799$ & $3,798,651$ \\
\hline N90 length (bp) & $3,001,655$ & $1,616,047$ \\
\hline Maximum length (bp) & $5,985,695$ & $7,438,647$ \\
\hline Minimum length (bp) & 61,794 & 95,361 \\
\hline $\mathrm{GC}$ content $(\%)$ & 53.33 & 53.65 \\
\hline Coverage between genomes (\%) & 90.07 & 93.48 \\
\hline \multicolumn{3}{|l|}{ Genome content } \\
\hline Protein coding genes & 10,773 & 10,517 \\
\hline BUSCO & $97.9 \%$ & $97.9 \%$ \\
\hline Length of genes (bp) & $19,057,009$ & $18,460,127$ \\
\hline Mean gene length (bp) & 1,769 & 1,755 \\
\hline Gene GC \% & 58.51 & 58.60 \\
\hline Exon number & 31,037 & 30,524 \\
\hline Coding gene with introns & 1.88 & 1.90 \\
\hline Mean exons per gene & 2.88 & 2.90 \\
\hline Mean exons length (bp) & 540 & 530 \\
\hline Intron number & 20,264 & 20,007 \\
\hline Mean intron length (bp) & 114 & 114 \\
\hline Mean intergenic length (bp) & 1,689 & 1,589 \\
\hline Number of transposons & 4,693 & 4,415 \\
\hline Total length of transposons (bp) & $5,024,491$ & $3,589,160$ \\
\hline Percentage of transposons in genome (\%) & 13.46 & 10.15 \\
\hline \multicolumn{3}{|l|}{ Genome annotation } \\
\hline Secretome & 798 & 793 \\
\hline CAZymes & 587 & 586 \\
\hline CAZymes (secreted) & 301 & 302 \\
\hline Protein kinases & 150 & 153 \\
\hline Transcription factors & 429 & 426 \\
\hline SCRPS & 303 & 299 \\
\hline
\end{tabular}

${ }^{a}$ CAZymes, carbohydrate-active enzymes; SCRPs, small cysteine-rich proteins; and BUSCO: benchmarking universal single-copy orthologs.

respectively (Table 1). Transposable elements were identified by de novo prediction, RepeatMasker (open 3.2.8, detailed parameters: -no_is, -norna, -engine, -s, -parallel=1, used Repbase version 15.08) and RepeatProteinMask (-noLowSimple, $-P$ value $=1 \mathrm{e}-4$, version open-4.0) (http:// www.repeatmasker.org). The analysis revealed that 13.46 and $10.15 \%$ of the S011 and S023 genomes, respectively, were composed of transposons.

The totality of de novo assembled draft genome was evaluated using BUSCO (benchmarking universal single-copy orthologs, fungi_odb10, version 3.1.0). Protein-coding genes in the S011 and S023 genomes were predicted using a combination of de novo-based GeneMark-ES (version Suite 4.10) (Borodovsky and Mclninch 1993), AUGUSTUS (version 2.6) (Stanke et al. 2008) and SNAP (version 11/29/2013) (Korf 2004) programs, as well as by the homology-based approaches previously described (Chen et al. 2018). The functional annotations of all genes were interrogated with known databases using BLASTP to identify homologs, including the databases of $n r$, eggNOGs (Powell et al. 2012), and InterProScan (Jones et al. 2014). The subcellular localization predictions were identified following procedures described previously (Klosterman et al. 2011). Putative secreted carbohydrate-active enzymes (CAZymes) were identified using the HMM-based routine of the carbohydrate-active-enzymes database (Huang et al. 2018).

Overall, the S011 and S023 genomes encoded 10,773 and 10,517 putative protein-encoding genes, respectively. Among these, 798 and 793 genes encoded secreted proteins in S011 and S023, respectively, and among these 303 and 299 belonged to the small cysteine-rich proteins (SCRPs, $<400$ amino acids, $\geq 4$ cysteine residues) (Table 1), respectively. A total of 587 and 586 genes that encode CAZymes were identified in S011 and S023, respectively. A total of 153 and 152 protein kinases were predicted by HMM searches locally with Kinomer version 1.0 

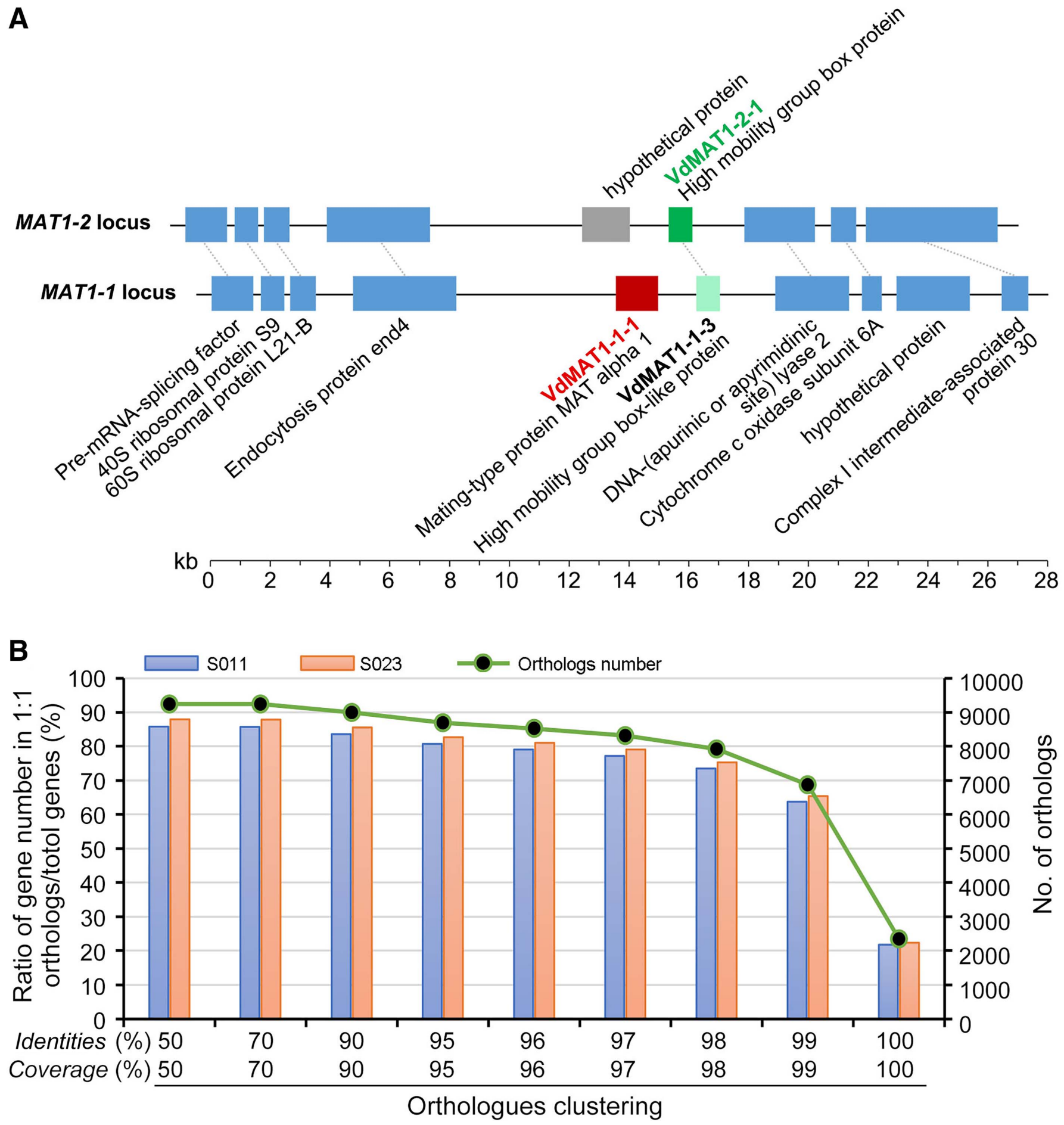

Fig. 1. Comparative genomics between strains S011 and S023 of Verticillium dahliae. A, Sketch map of the MAT1-1 and MAT1-2 loci in the sequenced genomes of $V$. dahliae strains S011 and S023. B, Homolog (1:1, equal copies between MAT1-1 and MAT1-2 genomes) comparisons between the S011 and S023 genomes. The homologs were clustered at identity and coverage levels: 50, 70, 90 , and 95 to $100 \%$.

(Martin et al. 2009), while 429 and 426 transcription factors were predicted using the InterPro database in S011 and S023 (Table 1), respectively. This information revealed that the genome composition, especially in the pathogenicity-related gene numbers, was highly similar in both genomes.

The mating-type loci of S011 and S023 were identified by the BLASTN using the MAT1-1-1 and MAT1-2-1 sequences (Usami et al. 2009). The MAT1-1 and MAT1-2 loci displayed high synteny in gene orthologs, and the MAT1-1 locus also contained the putative VdMAT1-1-3 that encodes the HMG homolog (Fig. 1A). However, the encoded genes were differentiated between the 
genomes of MAT1-1 and MAT1-2 idiomorphs. First, the size of these two genomes is different, and with 90.07 and $93.48 \%$ coverage when the short sequence reads from S023 or S011 were mapped to the S011 or S023 genome (Table 1), respectively. Homolog comparisons (parameters: both identities and coverage were set as up to $50,70,90$, and 95 to $98 \%$ ) between the two genomes revealed that the percent of homologs in two genomes were $>75 \%$, indicating that the genes are conserved between the MAT1-1 and MAT1-2 genomes. However, only around 20\% homologs were identical (at identity and coverage length of 100\%) between S011 and S023 genomes (Fig. 1B), indicating that minor sequence divergence is widespread among the genes in the MAT1-1 and MAT1-2 genomes.

In this study, high-quality genomic assemblies of V. dahliae MAT1-1 (S011) and MAT1-2 (S023) were obtained. Strains S011 and S023 were both derived from the same sunflower field. These resources will support our future genomic characterization of MAT1-1 V. dahliae strains, and the study of the mechanisms of sexual and asexual reproduction in $V$. dahliae. The status of reproductive mode in $V$. dahliae will expand our knowledge of the biology of Verticillium spp. and facilitate studies that seek to understand the evolutionary characteristics of fungal pathogens over complex and changeable environments.

This Whole Genome Shotgun project has been deposited at DDBJ/ENA/GenBank under the accessions JADFUI000000000 (S011) and JADFUJ000000000 (S023). The version described in this paper is version JADFUI000000000 and JADFUJ000000000. The BioProject accession is PRJNA666276.

\section{Literature Cited}

Atallah, Z. K., Maruthachalam, K., du Toit, L., Koike, S. T., Davis, R. M., Klosterman, S. J., Hayes, R. J., and Subbarao, K. V. 2010. Population analyses of the vascular plant pathogen Verticillium dahliae detect recombination and transcontinental gene flow. Fungal Genet. Biol. 47:416-422.

Atallah, Z. K., Maruthachalam, K., and Subbarao, K. V. 2012. Sources of Verticillium dahliae populations affecting lettuce. Phytopathology 102:1071-1078.

Atallah, Z. K., Maruthachalam, K., Vallad, G. E., Davis, R. M., Klosterman, S. J., and Subbarao, K. V. 2011. Analysis of Verticillium dahliae suggests a lack of correlation between genotypic diversity and virulence. Plant Dis. 95:1224-1232.

Baroudy, F., Putman, A. I., Habib, W., Puri, K. D., Subbarao, K. V., and Nigro, F. 2019. Genetic diversity of Verticillium dahliae populations from olive and potato in Lebanon. Plant Dis. 103:656-667.

Becker, K., Beer, C., Freitag, M., and Kück, U. 2015. Genome-wide identification of target genes of a mating-type $\alpha$-domain transcription factor reveals functions beyond sexual development. Mol. Microbiol. 96:1002-1022.

Boetzer, M., and Pirovano, W. 2014. SSPACE-LongRead: Scaffolding bacterial draft genomes using long read sequence information. BMC Bioinformatics 15:211.

Borodovsky, M., and Mclninch, J. 1993. GeneMark: Parallel gene recognition for both DNA strands. Comput. Chem. 17:123-133.

Chen, J. Y., Liu, C., Gui, Y. J., Si, K. W., Zhang, D. D., Wang, J., Short, D. P. G., Huang, J. Q., Li, N. Y., Liang, Y., Zhang, W. Q., Yang, L., Ma, X. F., Li, T. G., Zhou, L., Wang, B. L., Bao, Y. M., Subbarao, K. V., Zhang, G. Y., and Dai, X. F. 2018. Comparative genomics reveals cotton-specific virulence factors in flexible genomic regions in Verticillium dahliae and evidence of horizontal gene transfer from Fusarium. New Phytol. 217:756-770.

Chen, J. Y., Zhang, D. D., Huang, J. Q., Wang, D., Hao, S. J., Li, R., Puri, K. D., Yang, L., Tong, B. Z., Xiong, K. X., Simko, I., Klosterman, S. J., Subbarao, K. V., and Dai, X. F. 2020. Genome sequence of Verticillium dahliae race 1 isolate VdLs.16 from lettuce. Mol. Plant-Microbe Interact. 33:1265-1269.

Coppin, E., Debuchy, R., Arnaise, S., and Picard, M. 1997. Mating types and sexual development in filamentous ascomycetes. Microbiol. Mol. Biol. Rev. 61:411-428.

de Jonge, R., Bolton, M. D., Kombrink, A., van den Berg, G. C., Yadeta, K. A., and Thomma, B. P. 2013. Extensive chromosomal reshuffling drives evolution of virulence in an asexual pathogen. Genome Res. 23:1271-1282.

Depotter, J. R., Deketelaere, S., Inderbitzin, P., Tiedemann, A. V., Hofte, M., Subbarao, K. V., Wood, T. A., and Thomma, B. P. 2016. Verticillium longisporum, the invisible threat to oilseed rape and other brassicaceous plant hosts. Mol. Plant Pathol. 17:1004-1016.

Depotter, J. R., Shi-Kunne, X., Missonnier, H., Liu, T., Faino, L., van den Berg, G. C., Wood, T. A., Zhang, B., Jacques, A., Seidl, M. F., and Thomma, B. P.
2019. Dynamic virulence-related regions of the plant pathogenic fungus Verticillium dahliae display enhanced sequence conservation. Mol. Ecol. 28:3482-3495.

Dyer, P. S., Inderbitzin, P., and Debuchy, R. 2016. Mating-type structure, function, regulation and evolution in the Pezizomycotina. 351-385 in: Growth, Differentiation and Sexuality, The Mycota I. 3rd ed. J. Wendland, ed. Springer International Publishing, Switzerland.

Dyer, P. S., and O'Gorman, C. M. 2011. A fungal sexual revolution: Aspergillus and Penicillium show the way. Curr. Opin. Microbiol. 14:649-654.

English, A. C., Richards, S., Han, Y., Wang, M., Vee, V., Qu, J., Qin, X., Muzny, D. M., Reid, J. G., Worley, K. C., and Gibbs, R. A. 2012. Mind the gap: Upgrading genomes with Pacific Biosciences RS long-read sequencing technology. PLoS One 7:e47768.

Huang, L., Zhang, H., Wu, P., Entwistle, S., Li, X., Yohe, T., Yi, H., Yang, Z., and Yin, Y. 2018. dbCAN-seq: A database of carbohydrate-active enzyme (CAZyme) sequence and annotation. Nucleic Acids Res. 46:D516-D521.

Inderbitzin, P., Bostock, R. M., Davis, R. M., Usami, T., Platt, H. W., and Subbarao, K. V. 2011a. Phylogenetics and taxonomy of the fungal vascular wilt pathogen Verticillium, with the descriptions of five new species. PLoS One 6:e28341.

Inderbitzin, P., Davis, R. M., Bostock, R. M., and Subbarao, K. V. 2011b. The ascomycete Verticillium longisporum is a hybrid and a plant pathogen with an expanded host range. PLoS One 6:e18260.

Inderbitzin, P., Davis, R. M., Bostock, R. M., and Subbarao, K. V. 2013. Identification and differentiation of Verticillium species and $V$. Iongisporum lineages by simplex and multiplex PCR assays. PLoS One 8:e65990.

Jones, P., Binns, D., Chang, H. Y., Fraser, M., Li, W., McAnulla, C., McWilliam, H., Maslen, J., Mitchell, A., Nuka, G., Pesseat, S., Quinn, A. F., Sangrador-Vegas, A., Scheremetjew, M., Yong, S., Lopez, R., and Hunter, S. 2014. InterProScan 5: Genome-scale protein function classification. Bioinformatics 30:12361240.

Karapapa, V. K., Bainbridge, B. W., and Heale, J. B. 1997. Morphological and molecular characterization of Verticillium longisporum comb. nov., pathogenic to oilseed rape. Mycol. Res. 101:1281-1294.

Keszthelyi, A., Jeney, A., Kerenyi, Z., Mendes, O., Waalwijk, C., and Hornok, L. 2007. Tagging target genes of the MAT1-2-1 transcription factor in Fusarium verticillioides (Gibberella fujikuroi MP-A). Antonie van Leeuwenhoek 91:373-391.

Kistler, H. C., and Miao, V. P. 1992. New modes of genetic change in filamentous fungi. Annu. Rev. Phytopathol. 30:131-153.

Klix, V., Nowrousian, M., Ringelberg, C., Loros, J. J., Dunlap, J. C., and Pöggeler, S. 2010. Functional characterization of MAT1-1-specific mating-type genes in the homothallic ascomycete Sordaria macrospora provides new insights into essential and nonessential sexual regulators. Eukaryot. Cell 9:894-905. 
Klosterman, S. J., Subbarao, K. V., Kang, S., Veronese, P., Gold, S. E., Thomma, B. P., Chen, Z., Henrissat, B., Lee, H., Park, J., Garcia-Pedrajas, M. D., Barbara, D. J., Anchieta, A., de Jonge, R., Santhanam, P., Maruthachalam, K., Atallah, Z., Amyotte, S. G., Paz, Z., Inderbitzin, P., Hayes, R. J., Heiman, D. I., Young, S., Zeng, Q. D., Engels, R., Galagan, J., Cuomo, C. A., Dobinson, K. F., and $\mathrm{Ma}, \mathrm{L}$. J. 2011. Comparative genomics yields insights into niche adaptation of plant vascular wilt pathogens. PLoS Pathog 7:e1002137.

Korf, I. 2004. Gene finding in novel genomes. BMC Bioinformatics 5:59.

Martin, D. M. A., Miranda Saavedra, D., and Barton, G. J. 2009. Kinomer v1.0: A database of systematically classified eukaryotic protein kinases. Nucleic Acids Res. 37:D244-D250.

McCormick, R. F., Truong, S. K., and Mullet, J. E. 2015. RIG: Recalibration and interrelation of genomic sequence data with the GATK. G3 (Bethesda) 5:655-665.

Milgroom, M. G., Del Mar Jimenez-Gasco, M., Olivares-Garcia, C., and Jimenez-Diaz, R. M. 2016. Clonal expansion and migration of a highly virulent, defoliating lineage of Verticillium dahliae. Phytopathology 106:1038-1046.

Milgroom, M. G., Jimenez-Gasco, M. M., Olivares, G. C., Drott, M. T., and Jimenez-Diaz, R. M. 2014. Recombination between clonal lineages of the asexual fungus Verticillium dahliae detected by genotyping by sequencing. PLoS One 9:e106740.

Pöggeler, S. 2001. Mating-type genes for classical strain improvements of ascomycetes. Appl. Microbiol. Biotechnol. 56:589-601.

Powell, S., Szklarczyk, D., Trachana, K., Roth, A., Kuhn, M., Muller, J., Arnold, R., Rattei, T., Letunic, I., Doerks, T., Jensen, L. J., von Mering, C., and Bork, P.
2012. eggNOG v3.0: Orthologous groups covering 1133 organisms at 41 different taxonomic ranges. Nucleic Acids Res. 40:284-289.

Rafiei, V., Banihashemi, Z., Bautista-Jalon, L. S., del Mar Jiménez-Gasco, M., Turgeon, B. G., and Milgroom, M. G. 2018. Population genetics of Verticillium dahliae in Iran based on microsatellite and single nucleotide polymorphism markers. Phytopathology 108:780-788.

Seidl, M. F., and Thomma, B. P. 2014. Sex or no sex: Evolutionary adaptation occurs regardless. BioEssays 36:335-345.

Short, D. P., Gurung, S., Gladieux, P., Inderbitzin, P., Atallah, Z. K., Nigro, F., and Subbarao, K. V. 2015. Globally invading populations of the fungal plant pathogen Verticillium dahliae are dominated by multiple divergent lineages. Environ. Microbiol. 17:2824-2840.

Short, D. P. G., Gurung, S., Hu, X., Inderbitzin, P., and Subbarao, K. V. 2014. Maintenance of sex-related genes and the co-occurrence of both mating types in Verticillium dahliae. PLoS One 9:e112145

Stanke, M., Diekhans, M., Baertsch, R., and Haussler, D. 2008. Using native and syntenically mapped cDNA alignments to improve de novo gene finding. Bioinformatics 24:637-644.

Turgeon, B. G., and Yoder, O. C. 2000. Proposed nomenclature for mating type genes of filamentous ascomycetes. Fungal Genet. Biol. 31:1-5.

Usami, T., Itoh, M., and Amemiya, Y. 2008. Mating type gene MAT1-2-1 is common among Japanese isolates of Verticillium dahliae. Physiol. Mol. Plant Pathol. 73:133-137.

Usami, T., Itoh, M., and Amemiya, Y. 2009. Asexual fungus Verticillium dahliae is potentially heterothallic. J. Gen. Plant Pathol. 75:422-427. 